\title{
ASKING THE RIGHT QUESTIONS
}

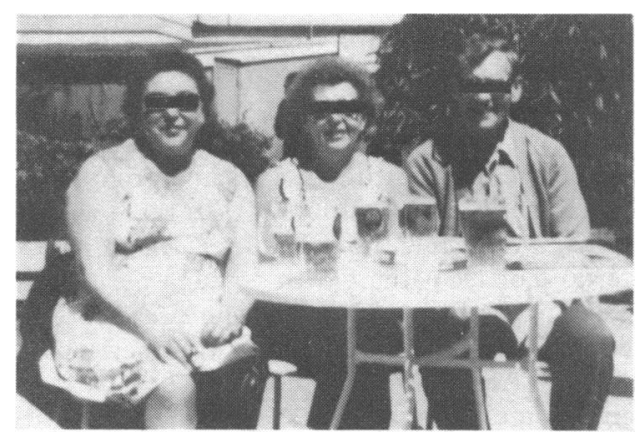

Do you smoke? What do you smoke? How much?

How long have you been smoking?

Do you drink? What do you drink?

Roughly how much?

How long have you been drinking ?

Do you take tablets or medicines? What are they for ?
Many people who are not concerned with alcoholism believe that it is impossible to get reliable information about drinking patterns. The reasons given are (a) self-deception and guilt on the part of the alcoholic; $(b)$ the difficulty in many cases of a heavy drinker, for example, a barman, being able to assess his intake; and (c) failure of doctors to take an adequate drinking history. Even so, $80 \%$ of alcoholics give a drinking history which tallies with that from other sources, and if quantities are accepted as approximations, valuable information can be obtained.

During history taking, questions about alcohol consumption should always be included (and recorded), both in hospital and in general practice. It is important that they be asked in the same neutral manner as other questions. They may be included with questions on symptoms or past illnesses which are relevant to the diagnosis or combined with questions on smoking and self-medication, after a review of the systems.

A positive response will allow the doctor to explore the pattern of drinking in greater detail. He can make a reasonably accurate assessment by asking what the patient has drunk in the previous week. If the answer is nothing the patient is unlikely to be an active alcoholic. If he denies drinking he should be asked why-he may abstain for religious or temperance reasons or because he is a former alcoholic.

If heavy drinking is suspected and the patient seems evasive, one technique is to use a facetious approach and to set the figure very highfor example with beer, "Thirty pints a day ?"-and gradually to reduce the quantities asked. The astute observer can usually gauge the approximate level if he watches the subject's reaction. Do not be misled by the individual who says he used to drink heavily but does not do so now. Do not ask a patient whether he is an alcoholic; he will almost certainly resent the question because it conflicts with his image of himself.

\section{Microcomputers and questionnaires}

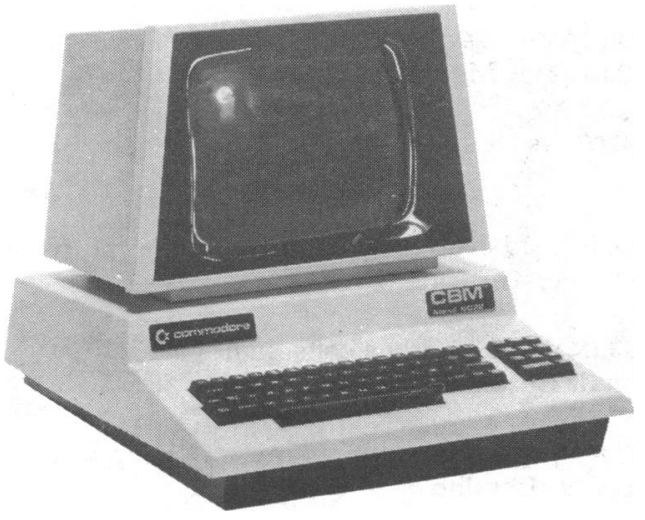

A new refinement has come with the advent of microcomputers. Programmes consisting of a series of questions on drinking habits, quantities consumed, and problems associated with heavy drinking have now been designed. They can be stored on floppy disks and used with several commercial microcomputers. The only major adaptation that needs to be made is the construction of a console (costing $£ 100-200)$ by which the patient keys in his replies. This approach has been successful in obtaining reproducible histories from patients with a wide range of educational backgrounds and intelligence.

For screening patients in hospital or general practice it is appropriate to establish the pattern of drinking and average daily alcohol consumption. "Quantity-frequency" questionnaires can be used as an aide memoire; self-administered versions are particularly useful when time is at a premium. They can also be given to the spouse. Questions are asked about the type of alcohol taken, for each type the frequency of drinking using fixed-choice categories, the usual quantity on each drinking day, and the maximum amount on any one occasion. The average daily consumption can then be calculated in grams of alcohol.

A simpler method is to use a drinking diary in which the patient is asked to note down every alcoholic drink he has taken in the previous seven days. This gives a reasonable approximation to intake as established by quantity-frequency questionnaires or by interview. 


\section{Questionnaires}

Several questionnaires have been developed for the diagnosis of alcoholism; the Michigan Alcoholism Screening Test (MAST) and the "CAGE" questionnaires have withstood the test of time. The MAST is available in two versions: the original 25-item questionnaire, which is administered by an interviewer, and a shorter, self-administered version comprising the 10 items of greatest discriminatory value. A score of five points or more on the Brief MAST is taken as diagnostic of alcoholism.

Both versions have been used successfully to identify alcoholics among general and psychiatric hospital patients. They are useful in screening programmes and for research, but in the clinical setting the shorter "CAGE" questionnaire, which

\section{Cage}

Have you ever felt you ought to cut down on your drinking ?

Have people annoyed you by criticising your drinking ?

Have you ever felt bad or guilty about your drinking?

Have you ever had a drink first thing in the morning to steady your nerves or get rid of a hangover? ("eye-opener")

From Maytield I), McLeod G, Hall P. Am f Psych 1974;131: $1121-3$.

\section{Brief MAST}

Circle correct answer

Do you feel you are a normal drinker? . . YES NO (2 pts)

Do friends or relatives think you are a normal drinker? .. $\quad . \quad \ldots$

Have you ever attended a meeting of Alcoholics Anonymous? .. . .

Have you ever lost friends or girlfriends or boyfriends because of drinking ?..

Have you ever got into trouble at work because of drinking? $\quad \ldots \quad \ldots \quad \ldots$ YES (2 pts) NO

Have you ever neglected your obligations, your family, or your work for two or more days in a row because you were drinking? YES (2 pts) NO

Have you ever had delirium tremens (DTs), severe shaking, heard voices or seen things that were not there after heavy drinking? YES (5 pts) NO

Have you ever gone to anyone for help about

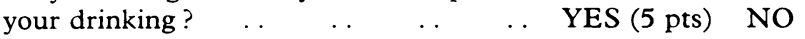

Have you ever been in a hospital because of drinking? $\begin{array}{llllllll} & \text {. } & \ldots & \ldots & \ldots & \ldots & \text { YES (5 pts) } & \text { NO }\end{array}$

Have you ever been arrested for drunken driving or driving after drinking ? . . YES (2 pts) NO

\section{Total score}

From Pokomy AD, Miller BA, Kaplan HB. Am f Psych 1972;129:342-5.

\section{Questionnaire for patient attending hospital or GP}

Do you drink alcohol? YES NO

If $\mathrm{NO}$, did you ever drink? . YES NO

How old were you when you started? (Age in years)

How often do/did you drink (Circle)

Daily/2-3 times a week/Once a week

Weekends only/Once a month/Less

What do you drink? (Circle more than one if necessary)

$$
\begin{aligned}
& \text { Beer-specify type (mild, bitter, lager, stout, etc) } \\
& \text { Spirits/Sherry/Wine/Cider } \\
& \text { Other-specify type (home brew, tonic wine, etc) }
\end{aligned}
$$

How much do you drink?

$$
\begin{array}{ll}
\text { Specify } & \text { pints of beer } \\
& \text { tots/measures of spirits or part of bottle } \\
\text { bottle } & \text { glasses of sherry, wine, cider or part of }
\end{array}
$$

Did you use to drink more than this regularly? . . YES NO

Have you ever deliberately cut down on your drinking ?. . consists of four items, is more appropriate. Two or more positive replies are said to identify problem drinkers. Neither test should be regarded as more than $75 \%$ accurate.

Alcoholism questionnaires have the disadvantage that they depend on honest replies from an individual who may be unwilling to admit to social problems or police convictions. They lack the inherent subtlety of the medical interview, and the self-administered versions are dependent on the patient's being well enough and sufficiently motivated to complete them. By concentrating on social problems and symptoms of alcohol dependence they tend to encourage a narrow concept of alcoholism. Patients with physical diseases caused by alcohol may never have suffered any of the social consequences of heavy drinking nor been dependent on alcohol. Indeed their drinking habits may have been regarded as socially quite acceptable, so that such patients will be missed by this type of questionnaire.

Dr A Paton, MD, FRCP, was formerly consultant physician, Dudley Road Hospital, Birmingham, and Dr J B Saunders, MB, MRCP (now lecturer, Liver Unit, King's College

Hospital, London) was Sheldon Clinical research fellow, Dudley Road Hospital, Birmingham.

The microcomputer was reproduced by permission of Commodore Ltd, Slough. 